gesunken. Nur die Lebensqualität, erfasst anhand des Childhood Asthma Questionnaire, und die Reaktion im Hautpricktest zeigten in der SLIT-Gruppe eine stärkere Verbesserung als in der Plazebogruppe. Parallel waren auch die IgE- und $\mathrm{IgG}_{4}$-Antikörpertiter in der SLIT-Gruppe stärker angesteigen als in der Plazebogruppe. Es traten lediglich leichte bis mittelschwere lokale Nebenwirkungen der SLIT auf, die passager waren.

Die Autoren führen die mangelnde Effektivität der SLIT in dieser Studie unter anderem auf die geringe Fallzahl - es waren mehr Teilnehmer geplant und den geringen Asthmaschweregrad der Kinder zurück. Zudem hatte das Design für beide Gruppen eine Optimierung der medikamentösen Therapie und der Milbenreduktion während der Studie vorgesehen, womit die signifikanten Verbesserungen auch in der Plazebogruppe zu erklären sein könnten.

Fazit: Bei Kindern mit leichtem bis mäßigem Asthma aufgrund einer Sensibilsi- sierung gegen Hausstaubmilben ist eine sublinguale Immuntherapie zusätzlich zu einer optimierten Therapie und einer möglichst effektiven Reduktion der Allergenbelastung nach dieser Studie nicht vorteilhaft.

Pham-Thi $\mathbf{N}$ et al. Assessment of sublingual immunotherapy efficacy in children with house dust mite-induced allergic asthma optimally controlled by pharmacologic treatment and mite-avoidance measures. Pediatr Allergy Immunol 2007; 18: 47-57

\section{Neue Cochrane-Metaanalyse zur spezifischen Immuntherpaie}

\author{
Die Cochrane Collaboration hatte bereits im Jahr 2003 Stellung \\ bezogen zum Nutzen der spezifischen sublingualen Immuntherapie \\ bei allergischer Rhinitis. Mit dreijähriger Verspätung folgte jetzt die \\ Metaanayse zur spezifischen subkutanen Hyposensibilisierung.
}

D as Reviewer-Team um Dr. Moisés Calderon, London, bezog in seine Metaanalyse 51 randomisierte doppelblinde plazebokontrollierte Studien ein, in denen Patienten mit diagnostisch verifizierter allergischer Rhinitis aufgrund einer Sensibilisierung gegen Baum-, Gräser- oder Kräuterpollen mit einer subkutanen Monoallergen-Immuntherapie - optimaler Majorallergengehalt zwischen 5 und $20 \mu \mathrm{g} /$ Injektion - oder Plazeboinjektionen behandelt worden waren. Damit standen die Daten von 2.871 Patienten - 1.645 unter Verum, 1.226 unter Plazebo - zur Verfügung, die im Durchschnitt 18 Injektionen erhalten hatten.

Bei den verwertbaren Symptomscores aus 15 Studien ergab sich insgesamt eine deutliche Symptomreduktion in der Gruppe mit Immuntherapie ( $\mathrm{p}$ 0,00001) im Vergleich zu Plazebo. Auch bei den 13 Studien, bei denen die Medikationsscores ausgewertet werden konnten, zeigte sich eine deutliche Überlegenheit der Verumtherapie im Vergleich zu Plazebo ( $\mathrm{p}<0,00001)$. Die klinische Interpretation der errechneten Effektgröße ist allerdings schwierig.

Eine Adrenalininjektion aufgrund schwerer systemischer Nebenwirkungen war in den Verumgruppen bei 19 von insgesamt 14.085 Injektionen erforderlich $(0,13 \%)$, in den Gruppen, die Plazebo erhielten, bei einer von 8.278 Injektionen $(0,01 \%)$. Todesfälle traten nicht auf. Angaben über den klinischen Hintergrund der Patienten wurden in den Publikationen nicht gemacht. Aus anderen Studien weiß man aber, dass schwere Nebenwirkungen fast ausschließlich bei Patienten mit koexistierendem Asthma auftreten, häufig haben die Patienten noch weitere Begleiterkrankungen wie Diabetes oder kardiovaskuläre Risikofaktoren.

Immunologische Parameter wurden ebenfalls in einigen der Studien untersucht. Zwar waren die Ergebnisse im Detail heterogen, doch ergab sich sowohl für das spezifische IgG als auch für das spezifische $\operatorname{IgG}_{4}$ insgesamt ein signifikanter Anstieg bei den aktiv behandelten Patienten. Eindeutige Aussagen zum spezifischen IgE konnten nicht gemacht werden. Die Relevanz der immunologischen Veränderungen beim IgG ist noch nicht klar. In der Praxis könnten aber möglicherweise die IgG-Veränderungen als Prädiktoren für das Ansprechen bzw. den klinischen Verlauf der Immuntherapie herangezogen werden.

\section{Die Macht der Metaanalyse \\ Die Cochrane Colla- boration ist ein 1993 gegründetes inter- nationales Netz von Wissenschaftlern und Ärzten, das zum \\ Ziel hat, durch systematische Meta- analysen die Evidenz medizinischer Therapien zu bewerten. Der Name der Organisation geht zurück auf den schottischen Epidemiologen Sir Archibald Leman Cochrane, der als Begründer der evidenzbasierten Medizin gilt. Die von den weltweit etwa 50 Cochrane Review Groups erarbeiteten Analysen werden zentral in der Cochrane Library abgelegt. Sie bieten Ärzten eine wissenschaftlich fundierte Informationsgrundlage, um den aktuellen Stand der Forschung beur- teilen zu können. Die Library ist online unter www.thecochranelibrary.com recherchierbar, weitere Informationen bietet unter www.cochrane.de auch das Deutsche Cochrane-Zentrum in Freiburg.}

Fazit: Die spezifische subkutane Immuntherapie reduziert bei Patienten mit einer saisonalen allergischen Rhinitis signifikant Symptom- und Medikationsscores, so das Ergebnis einer Metaanalyse von 51 randomisierten doppelblinden plazebokontrollierten Studien. Schwere Nebenwirkungen treten nur selten auf und führen zu keiner langfristigen $\mathrm{Be}$ einträchtigung.

Calderon $\mathbf{M}$ et al. Allergen injection immunotherapy for seasonal allergic rhinitis. Cochrane Database Syst Rev. 2007; 24:CDoo1936 